\title{
Template Stripping of Perovskite Thin Films for Dry Interfacing and Surface Structuring
}

\section{Journal Article}

\section{Author(s):}

Hernández Oendra, Alexander C.; De Leo, Eva; Koepfli, Stefan M. (D); Winkler, Jan M.; Rossinelli, Aurelio (D); Riedinger, Andreas; Norris, David J.; Prins, Ferry

Publication date:

2020-06-10

Permanent link:

https://doi.org/10.3929/ethz-b-000421265

Rights / license:

In Copyright - Non-Commercial Use Permitted

Originally published in:

ACS Applied Materials \& Interfaces 12(23), https://doi.org/10.1021/acsami.0c04006

Funding acknowledgement:

339905 - Quantum-Dot Plasmonics and Spasers (EC) 


\section{Template-Stripping of Perovskite Thin Films for Dry-Interfacing and Surface-Structuring}

Alexander C. Hernandez Oendra, Eva De Leo, Stefan M. Koepfli ${ }^{\S}$, Jan M. Winkler, Aurelio A. Rossinelli, Andreas Riedinger ${ }^{\dagger}$, David J. Norris, and Ferry Prins ${ }^{\dagger *}$

Optical Materials Engineering Laboratory, Department of Mechanical and Process Engineering, ETH Zurich, Rämistrasse 101, 8092 Zürich, Switzerland

\section{KEYWORDS}

perovskite, template-stripping, thin film, interface, flexibility

* To whom correspondence should be addressed: ferry.prins@uam.es 


\begin{abstract}
Combining excellent optoelectronic properties with the benefits of solution processability, metalhalide perovskites are promising materials for photovoltaic and light-emitting technologies. To facilitate the integration of perovskite thin films into optoelectronic devices, a need exists for simple and efficient fabrication methods. Here we present a template-stripping technique to produce ultra-flat and flexible perovskite thin films. We apply a one-step spin-coating procedure to produce high-quality $\mathrm{CH}_{3} \mathrm{NH}_{3} \mathrm{PbBr}_{3}$ perovskite thin films on top of ultra-flat silicon templates. These films can be mechanically cleaved from the template using a polymer adhesive to reveal the ultra-flat perovskite surfaces. We demonstrate how the flatness and flexibility of the templatestripped films enable new processing strategies based on dry interfacing, by interfacing them with plasmonic hole arrays. Moreover, we show that by using pre-patterned silicon templates, structured perovskite surfaces can be produced with feature sizes down to a micrometer.
\end{abstract}




\section{Introduction}

Metal-halide perovskites with chemical formula $\mathrm{CH}_{3} \mathrm{NH}_{3} \mathrm{PbX}_{3}(\mathrm{X}=\mathrm{I}, \mathrm{Br}$ or $\mathrm{Cl})$ have emerged as a versatile class of materials for optoelectronic devices thanks to several unique characteristics, including broadband-tunable optical band gaps, high ambipolar charge-carrier mobility and high defect tolerance. ${ }^{1-4}$ These materials have been efficiently incorporated as the active layer in photovoltaic devices and light-emitting diodes (LEDs). ${ }^{5,6}$ Additionally, perovskites benefit from the advantages of solution processability, potentially yielding more cost-efficient production compared to traditional semiconductor materials. Common fabrication methods include spincoating, vapor deposition, and solution-based crystal growth. ${ }^{7-9}$ The main challenge hampering their broader applicability is their environmental instability, both complicating their processing and limiting the durability of perovskite-based devices. ${ }^{10}$

The environmental instability of perovskites is particularly evident in their moisture sensitivity and high solubility in polar solvents, ${ }^{11}$ making post-processing of perovskite films incompatible with wet-chemistry approaches. The development of dry processing strategies, inspired for example by transfer printing ${ }^{12}$ or wafer-bonding techniques, ${ }^{13,14}$ may facilitate the realization of more complex device architectures.

Here, we present a template-stripping technique to produce ultra-flat and flexible $\mathrm{CH}_{3} \mathrm{NH}_{3} \mathrm{PbBr}_{3}$ perovskite thin films suitable for large-scale transfer printing. Template-stripping relies on the mechanical cleaving of thin films from ultra-flat silicon templates, exposing a thin-film surface of superior flatness. Originally developed for metallic thin films, ${ }^{15,16}$ the method has more recently been used to produce ultra-flat surfaces of solution processable materials, such as thin films of colloidal quantum dots. ${ }^{17}$ Template-stripping can be extended to allow the fabrication of ultra-flat thin films on flexible substrates, ${ }^{17,18}$ producing structures suitable for transfer printing. ${ }^{19}$ In this 
work, we adapt the template-stripping method to produce ultra-flat and flexible perovskite thin films. These structures can be transferred to various surfaces through dry interfacing, which we demonstrate by placing a perovskite thin film on top of a plasmonic hole array. We show that the resulting high-quality interface leads to plasmon enhanced outcoupling of fluorescent emission from the perovskite film. Finally, we demonstrate that by using pre-patterned silicon templates, we can generate surface-structured perovskite films with feature sizes as small as one micrometer.

\section{Results and Discussion}

Our template-stripping method for perovskite thin films is schematically presented in Figure 1a. In short, a thin film of $\mathrm{CH}_{3} \mathrm{NH}_{3} \mathrm{PbBr}_{3}$ perovskite is deposited onto a silicon template under inert atmosphere using a one-step spin-coating procedure, combined with the anti-solvent dripping method as described by Chen et al. ${ }^{20}$ After spin-coating, the film, which is around $170 \mathrm{~nm}$ thick, is annealed for 10 minutes at $100{ }^{\circ} \mathrm{C}$ to remove any residual solvents and then taken out of the glovebox for template-stripping. For this, a high viscosity UV-curable epoxy adhesive with fast curing time is used to attach a glass slide to the back of the film. Using a razor blade, the perovskite/epoxy/glass stack is mechanically cleaved from the template exposing the templatestripped perovskite surface. In an optional final step, the epoxy can be detached from the glass slide backing using a razor blade, yielding a free-standing and flexible perovskite-epoxy film as shown in Figure $\mathbf{1 b}$. 


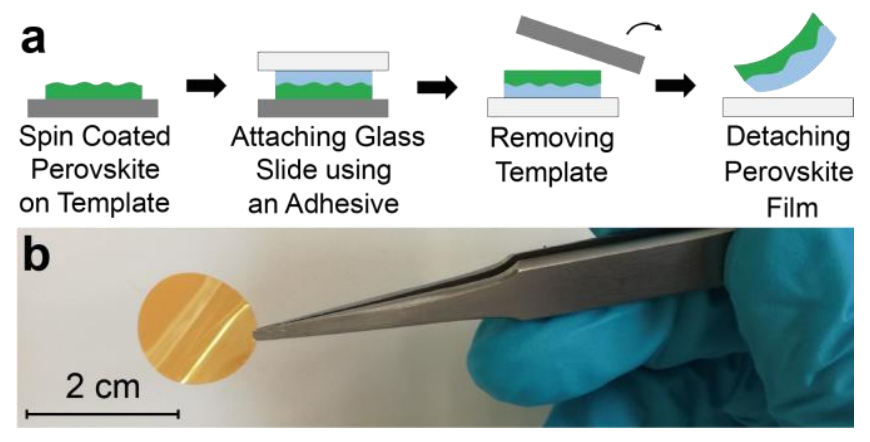

Figure 1. Fabrication of perovskite films. (a) Schematic depiction of the template-stripping process. (b) Image of free-standing and flexible perovskite-epoxy sample.

To characterize the flatness of our perovskite films, which is crucial for successful dry interfacing, we compared the spin-coated (Figure 2a) and template-stripped surfaces (Figure 2b) using atomic force microscopy (AFM). With the exception of isolated small-scale indentations, template-stripped perovskite films display a significantly reduced surface roughness with respect to the spin-coated surfaces. The observed small-scale indentations in the template-stripped films are likely the result of stresses introduced into the film during the annealing step, since they are absent in unannealed template-stripped films (see Figure S3). To quantify the overall flatness of the film, we determine the root mean square (RMS) roughness of $7 \times 4 \mu \mathrm{m}^{2}$ template-stripped and spin-coated perovskite surfaces. While the roughness of the spin-coated sample (RMS $=9.1 \mathrm{~nm}$ ) is comparable to previous perovskite thin-film measurements, ${ }^{21,22}$ a four-fold reduction in roughness is obtained using template-stripping $(\mathrm{RMS}=2.1 \mathrm{~nm}$ ). Importantly, the roughness of the template-stripped surface is also a factor two smaller than typically reported for thin films fabricated through vapor deposition $(\mathrm{RMS} \approx 5 \mathrm{~nm}) .{ }^{7,23,24}$ 


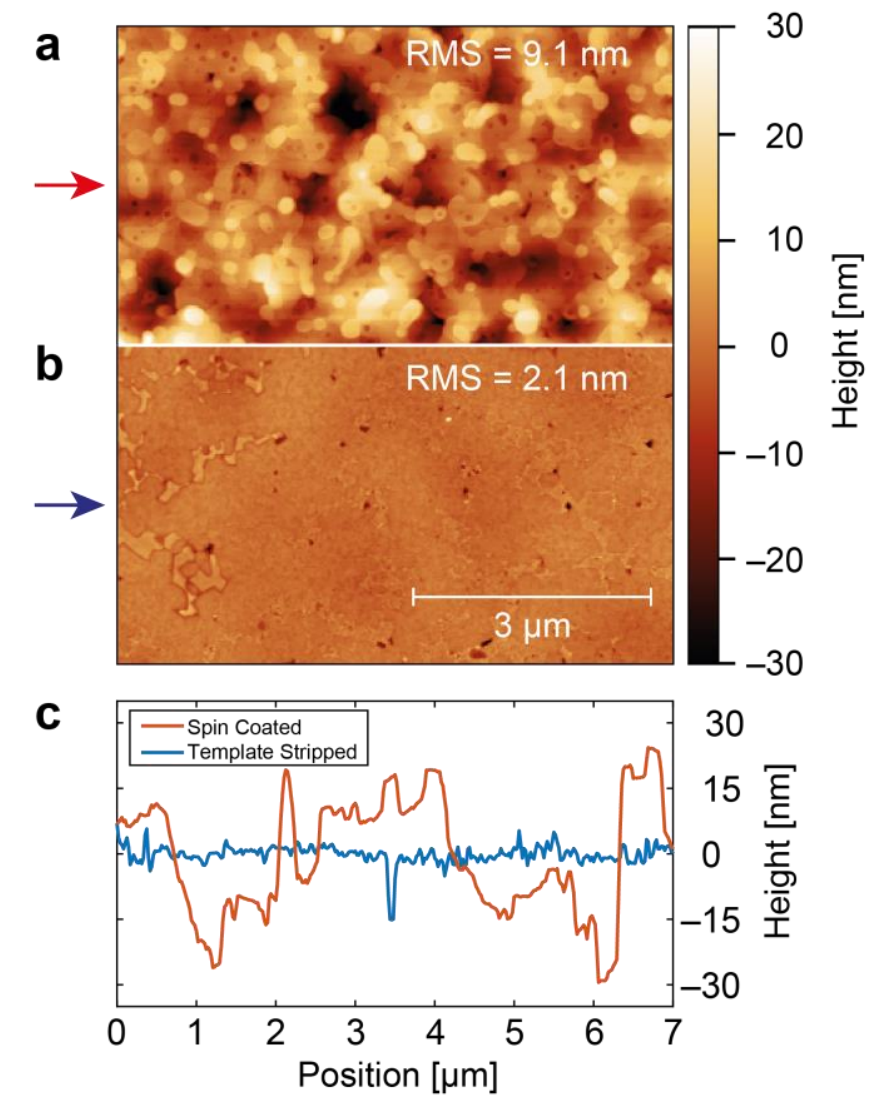

Figure 2. Surface roughness measurements of the fabricated perovskite films. (a, b) AFM measurements of $7 \times 4 \mathrm{~m}^{2}$ areas taken from the (a) spin-coated and (b) template-stripped side of a $\mathrm{CH}_{3} \mathrm{NH}_{3} \mathrm{PbBr}_{3}$ perovskite film. The measured root mean square roughness of the surfaces was $9.1 \mathrm{~nm}$ and $2.1 \mathrm{~nm}$, respectively. (c) Height profiles of the spin-coated (red) and template-stripped (blue) surfaces extracted from (a) and (b) at the positions indicated by the arrows for the full $7 \mu \mathrm{m}$ length.

The enhanced flatness of the perovskite film in combination with the flexibility of the polymer backing, opens up the possibility to interface the perovskite film with other structures. This can be of particular interest in applications whose performance relies significantly on the quality of their 
surfaces and interfaces such as plasmonics and photovoltaics. ${ }^{25,26}$ To demonstrate the benefits of our fabrication method, we interfaced $\mathrm{CH}_{3} \mathrm{NH}_{3} \mathrm{PbBr}_{3}$ thin films with metallic hole arrays designed to provide plasmon enhanced outcoupling of the fluorescent emission of our perovskite film. ${ }^{19,27}$ The hole-array samples were fabricated by evaporating an optically thick layer of silver ( $200 \mathrm{~nm})$ on pre-patterned silicon templates (see SI for detailed description) and used without any further modifications. The interfacing was performed by pressing a free-standing perovskite-epoxy film onto the silver hole array until the perovskite film becomes transparent and does not show any signs of interference fringes anymore. Figures 3a-c show images of eight silver hole arrays before, during, and after the interfacing procedure. The interference fringes (visible on the right side of Figure 3b) indicate the front where the flexible substrate slowly adheres to the hole array. As the front continues, the hole arrays become visible and the interference fringes disappear, indicating direct contact of the two surfaces over the hole arrays. In addition, the obvious color change of the hole array upon interfacing, caused by the change in the effective refractive index at the surface of the silver (going from low index air to high index perovskite film) ${ }^{28}$ is another indication of the direct contact between two surfaces.

Figure 3d shows the fluorescence micrograph corresponding to the fully interfaced perovskite structure of Figure 3c. While fluorescence is observed from the whole film, brighter emission is generated in the perovskite areas which are in direct contact with the hole array. As commonly occurs in high-refractive-index materials, a significant portion of the light generated inside a perovskite thin film undergoes total internal reflection when reaching the perovskite-air interface, generating waveguided modes that limit the overall brightness of the film. Plasmonic hole arrays can be used to redirect waveguided emission and enhance the outcoupling efficiency. ${ }^{29,30}$ 
To confirm that the enhanced brightness is indeed a result of matching the wavelength at which surface plasmons couple to light at normal incidence to the emission of the perovskite, we perform momentum-resolved spectroscopy. In Figure 3e, we present momentum-resolved reflectance for a hole array interfaced with the perovskite film. As expressed by the Bragg equation, a plasmonic hole array with a given pitch $\Lambda$ enhances the outcoupling of light at angle $\theta:{ }^{27}$

$$
k_{0} \sin (\theta)= \pm \frac{2 \pi n_{\mathrm{eff}}}{\lambda} \pm m \frac{2 \pi}{\Lambda}
$$

where $k_{0}$ is the wavevector of the outcoupled light, $n_{\text {eff }}$ is the effective refractive index of the surface plasmon mode, $\lambda$ is the wavelength of the outcoupled light, and $m$ is the diffraction order. The dashed lines in Figure 3e represent the solutions to the Bragg equation for $\Lambda=240 \mathrm{~nm}$, representing the experimental pitch (details of the fitting procedure can be found in the Supporting Information). The periodicity of this hole array is chosen to achieve good overlap between the crossing point of the surface plasmon dispersion branches, where outcoupling occurs at $\theta=0$, and the emission wavelength of the perovskite $\left(\lambda_{\mathrm{em}}=535 \mathrm{~nm}\right)$, as shown in Figure $\mathbf{3 f}$ in which the dispersion branches (dashed lines) are overlaid with the momentum-resolved emission spectrum. To quantify the enhancement of outcoupling under normal incidence, we compare the emission spectrum of the resonant $\Lambda=240 \mathrm{~nm}$ hole-array structure with off-resonant hole arrays, as shown in Figure 3g. The spectra are extracted by averaging the horizontal centerlines of the dispersed kspace measurements, which corresponds to a spectral emission measurement taken with an objective with numerical aperture (NA) of 0.04 . The integrated emission intensity shows that a six-fold enhancement of outcoupling intensity is obtained with respect to a fully off-resonant periodicity $(\Lambda=200 \mathrm{~nm})$. 

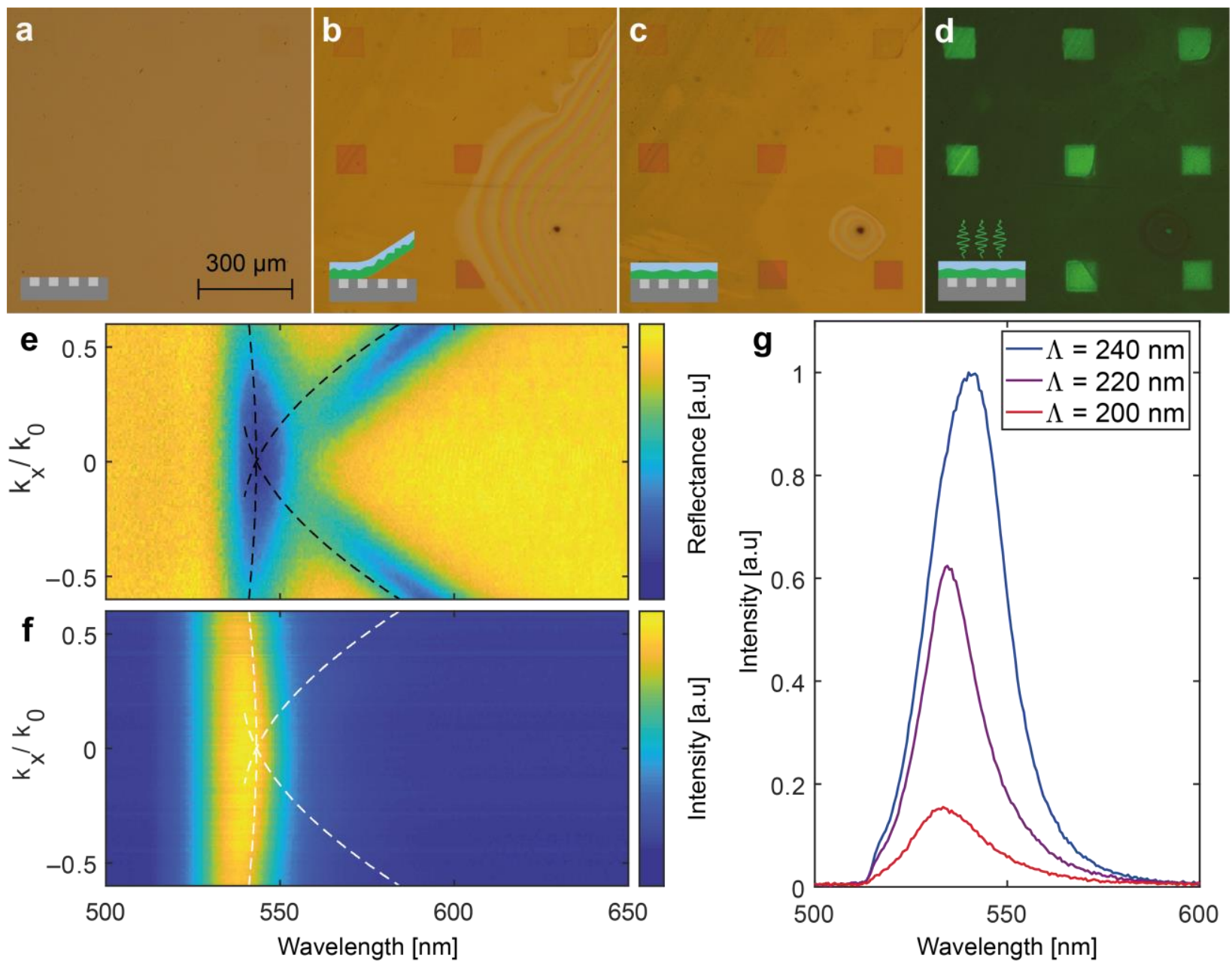

Figure 3. Interfacing perovskite films with hole arrays. (a-c) Sequential bright-field microscopy images of the interfacing procedure for an area with eight individual square hole arrays, with each array measuring $100 \times 100$ micrometer in size. The sequence starts with a plain silver hole array film (a), followed by a half-interfaced perovskite film on top of the silver hole arrays (b) and a fully interfaced structure (c). (d) Corresponding fluorescence image of the fully interfaced structure. (e, f) Dispersed k-space of the reflection and emission of an interfaced perovskite film on a $240 \mathrm{~nm}$ pitch hole array. The dashed lines in the dispersed k-space images are a guide-to-theeye and approximate the branches of the surface plasmon dispersion relation (see text for details). (g) Emission spectra from the perovskite film in contact with three different hole arrays of 
periodicity $\Lambda=200 \mathrm{~nm}, 220 \mathrm{~nm}$, and $240 \mathrm{~nm}$, extracted from the respective dispersed k-space measurements around $k_{x} / k_{0}=0$, corresponding to a collection cone with an NA of 0.04 .

Finally, we demonstrate that our template-stripping method can be employed to produce perovskite thin films with structured surfaces. Surface patterning using template-stripping has been demonstrated in the past for metallic thin films and colloidal assemblies. ${ }^{16,17}$ By substituting the flat silicon templates with pre-patterned silicon, structured features can be transferred to the template-stripped film (Figure 4a). To demonstrate this fabrication process for our perovskite films, we prepared patterned silicon templates using a combination of electron-beam lithography and reactive ion etching. Next, we functionalized the silicon templates with trichlorosilane based self-assembled monolayer to reduce the surface energy of the silicon surface (see SI for full experimental details). This step is required to reduce the adhesion between the silicon surface and the perovskite film during template-stripping, ensuring high-quality pattern transfer. Figure $\mathbf{4 b}$ shows a scanning electron micrograph of a patterned perovskite film. The smallest features on the structured surface are $1 \mu \mathrm{m}$ in size. We also achieved the fabrication of structures with submicrometer-sized features, but the yield of such small features is generally lower (see Figure S4). The limiting factor in achieving smaller features is the trade-off between wettability of the template during precursor deposition, requiring high surface energy, and the ability to strip the film without leaving behind any residuals, requiring a low surface energy. This balance is more critical for structured features than for flat surfaces, as previously observed for other materials. ${ }^{17,31}$

While perovskite films with surface structures have been produced in the past, the processing mostly relied on employing high pressure imprinting procedures or chemical vapor deposition techniques. ${ }^{32-35}$ More recently, low temperature thermal nanoimprinting lithography techniques 
have been reported, describing the fabrication of perovskite films with nanometer-sized features. ${ }^{36}$ This lead to the fabrication of high quality resonators with smooth surfaces, which significantly lowered the threshold for lasing condition. ${ }^{37}$ In addition, controlling the crystallization of perovskite films with soft lithography approaches utilizing only low pressure in their fabrication procedure has been reported. ${ }^{38}$ Another successful approach is the fabrication of structured perovskite films for single-mode microlasers via laser ablation..$^{39}$ Our template-stripping technique represents a versatile alternative and cost-effective strategy for surface structuring, which could be interesting for applications where using high pressures would be detrimental. Furthermore, the reusability of the patterned templates facilitates the production of many identical samples.

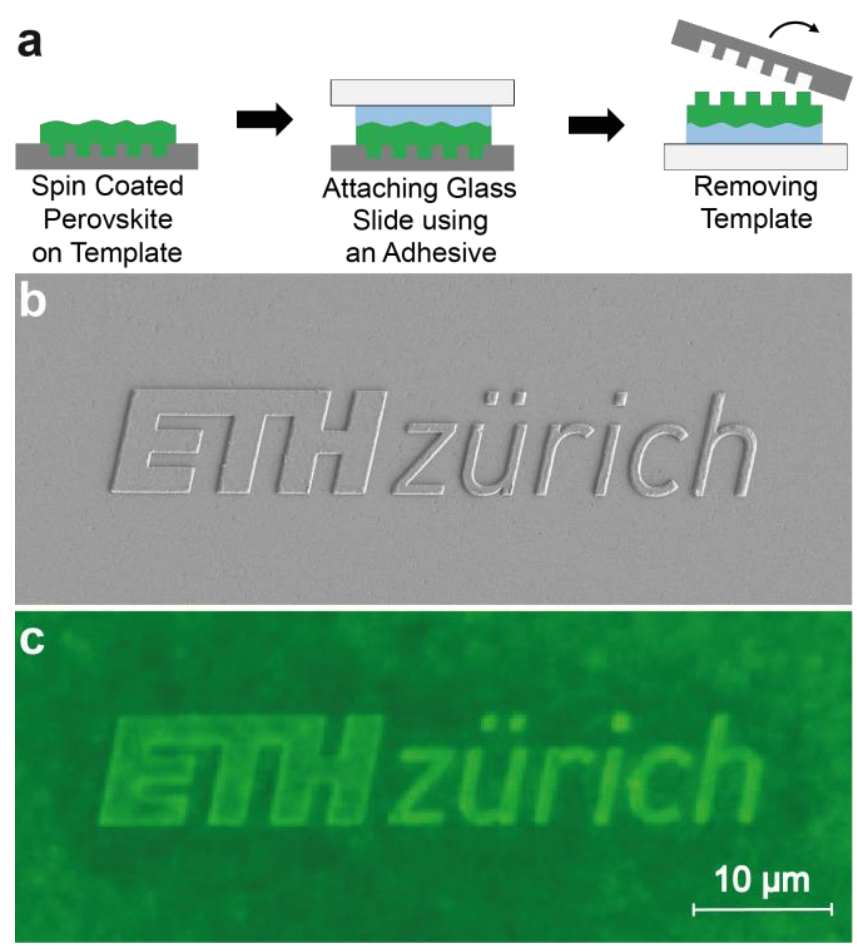

Figure 4. Template-stripping of structured perovskite films. (a) Schematics of the templatestripping process using pre-patterned silicon templates. (b) Scanning electron micrographs of an 
$\mathrm{CH}_{3} \mathrm{NH}_{3} \mathrm{PbBr}_{3}$ perovskite film featuring an ETH logo on its surface. Smallest feature size is $1 \mu \mathrm{m}$. (c) Fluorescence image of the same film.

\section{Conclusion}

In this work we have presented a template-stripping technique to produce smooth and flexible metal-organic halide perovskite thin films. We have demonstrated how, taking advantage of this simple and versatile technique to achieve high-quality film properties, dry-interfacing with more complex structures is possible. As an example of such a dry-interfaced structure, we have shown that the emission from the perovskite thin film can be enhanced by coupling it to plasmonic hole arrays where high quality interfaces are essential. We have also shown that by using pre-patterned templates our fabrication method can produce structured perovskite surfaces with micrometersized features. This fabrication technique could improve existing perovskite-based applications and enable future integration of metal organic halide perovskite materials into optoelectronic or photonic devices where solution-based approaches are not viable.

\section{MATERIALS AND METHODS}

Materials. Lead bromide (98\%, \#211141), lead iodide (99\%, \#211168), methylammonium bromide (>99\%, \#806498), methylammonium iodide (98\%, \#793493), sulfuric acid (95.0-97.0\%, \#320501), hydrochloric acid (37\%, \#258148), ethanol ( $\geq 99.8 \%$, \#02858), toluene (99.8\%, \#244511), dimethyl sulfoxide (DMSO, >99.5\%, \#D4540), octenyltrichlorosilane (96\%, \#539279), carbon tetrachloride (99,9\%, \#319961), bicyclohexyl (99\%, \#D79403), potassium permanganate $(\geq 99.0 \%, \# 223468)$, sodium periodate $(\geq 99.8 \%$, \#311448), potassium carbonate ( $\geq 99.0 \%$, \#P5833), sodium bisulfite ( 40\%, \#13438), and n-methyl-2-pyrrolidone (NMP, $\geq 99 \%$, \#M78259) 
were purchased from Sigma-Aldrich. Hydrogen peroxide (30\%,\#23615.248) was purchased from VWR. All chemicals and precursors were used as received. Deionized water (Milli-Q Advantage A10, Merck Millipore) was used throughout all steps.

Preparation of perovskite thin films. Silicon substrates were first immersed into piranha solution (1:1 ration of sulfuric acid and hydrogen peroxide, Caution: Piranha solution reacts violently with solvents and other organic material) for 15 min and subsequently treated with an ultraviolet-ozone cleaner (PSD Novascan) for $30 \mathrm{~min}$ to ensure a contamination-free surface prior to the perovskite thin-film deposition. The perovskite was deposited with a one-step spin-coating technique using a $25 \mathrm{wt} \%$ equimolar solution of lead bromide and methylammonium bromide in DMSO. ${ }^{20}$ The whole deposition was performed under inert atmosphere in a $\mathrm{N}_{2}$ glovebox. The spin-coating step was performed by pipetting $50 \mu 1$ onto the cleaned silicon substrate and spinning the solution at $2000 \mathrm{rpm}$ for $60 \mathrm{~s}$. After $37 \mathrm{~s}, 150 \mu \mathrm{l}$ of toluene was dripped onto the spinning sample, resulting in around $170 \mathrm{~nm}$ thin perovskite films. A schematic depiction of the entire spin-coating procedure and more detailed description of the fabrication procedure can be found in section S1 of the Supporting Information.

Template-stripping. The samples were taken out of the glovebox for template-stripping and the procedure was performed under ambient conditions. To expose the surface of the perovskite thin film, which is in contact with the silicon template, a drop of a fast-curing UV epoxy adhesive (Norland Optical Adhesive 63) was placed on the sample, and a glass slide, which was previously cleaned by sonication in isopropanol, was pressed onto it. The silicon/perovskite/epoxy/glass stack was then exposed to a $365 \mathrm{~nm}$ UV lamp and the adhesive was cured for $10 \mathrm{~min}$. The use of a fastcuring UV epoxy is essential to prevent prolonged exposure of the perovskite film to the liquid epoxy and the UV irradiation. In a next step, the glass backing was then separated from the 
template by placing the sample stack with the silicon template facing upwards and clipping off the template by applying pressure to its side using a razor blade. The silicon templates could be reused by removing the perovskite residue with DMSO and restarting with the cleaning procedure as stated in the previous section.

Experimental setup. Atomic force microscopy (AFM) measurements were performed on a Bruker Dimension FastScan and scanning electron microscopy (SEM) images were taken on a Hitachi S-4800. The dispersed k-space measurements were collected on a Ti-U inverted microscope (Nikon), using a high-magnification objective $(40 \mathrm{x}, \mathrm{NA}=0.6)$. The sample was illuminated with a halogen lamp (Nikon, LHS-H100C-1) to obtain reflection measurements. A 488 nm laser (Coherent, OBIS 488-100 LS) was used to excite the perovskite film for the fluorescence measurements. The reflected/emitted light is sent through one of the exit ports of the microscope after which it passes through a custom set of lenses and mirrors ${ }^{19}$ such that the back-focal-plane of the objective is imaged onto the entrance slit of a spectrograph (Acton SP2358, Princeton Instruments) and its dispersed values are imaged onto the attached CCD camera (PIXIS 256E, Princeton Instruments). The reflection measurements were normalized to the reflection by a plane silver surface. Optical characterization was performed under ambient conditions.

\section{Acknowledgements}

This work was supported by a Swiss National Science Foundation Ambizione Grant (FP). In addition, we acknowledge funding from the European Research Council under the European Union's Seventh Framework Program (FP/2007-2013)/ERC Grant 339905 (QuaDoPS Advanced 
Grant). We thank S. Meyer, Y. Fedoryshyn, A. Olziersky and U. Drechsler, for assistance in the fabrication.

\section{Notes}

The authors declare no competing financial interests.

\section{ASSOCIATED CONTENT}

\section{Supporting Information}

The Supporting Information is available free of charge.

Figure S1-S7. Further details to the fabrication of the perovskite films; XRD measurements; the functionalization of the surface of the templates; defects seen on the perovskite films; templatestripping of submicrometer-sized features; fabrication of plasmonic hole arrays and modelling their dispersion curves; analysis of halide diffusion over interface.

\section{AUTHOR INFORMATION}

\section{Corresponding Author}

* E-mail: ferry.prins@,uam.es (FP)

\section{Present Addresses}

§SK: Institute of Electromagnetic Fields (IEF), ETH Zurich, 8092 Zürich, Switzerland

\$AR: Max-Planck-Institute for Polymer Research, Ackermannweg 10, 55128 Mainz, Germany 
$\dagger$ FP: Condensed Matter Physics Center (IFIMAC), Universidad Autónoma de Madrid, 28049

Madrid, Spain

\section{REFERENCES}

(1) Herz, L. M. Charge-Carrier Dynamics in Organic-Inorganic Metal Halide Perovskites. Annu. Rev. Phys. Chem. 2016, 67, 65-89.

(2) Filip, M. R.; Eperon, G. E.; Snaith, H. J.; Giustino, F. Steric Engineering of Metal-Halide Perovskites with Tunable Optical Band Gaps. Nat. Commun. 2014, 5, 5757.

(3) Huang, H.; Bodnarchuk, M. I.; Kershaw, S. V.; Kovalenko, M. V.; Rogach, A. L. Lead Halide Perovskite Nanocrystals in the Research Spotlight: Stability and Defect Tolerance. ACS Energy Lett. 2017, 2, 2071-2083.

(4) Miyata, K.; Meggiolaro, D.; Trinh, M. T.; Joshi, P. P.; Mosconi, E.; Jones, S. C.; De Angelis, F.; Zhu, X.-Y. Large Polarons in Lead Halide Perovskites. Sci. Adv. 2017, 3, e1701217.

(5) Stranks, S. D.; Snaith, H. J. Metal-Halide Perovskites for Photovoltaic and Light-Emitting Devices. Nat. Nanotechnol. 2015, 10, 391-402.

(6) Green, M. A.; Ho-Baillie, A.; Snaith, H. J. The Emergence of Perovskite Solar Cells. Nat. Photonics 2014, 8, 506-514.

(7) Ono, L. K.; Leyden, M. R.; Wang, S.; Qi, Y. Organometal Halide Perovskite Thin Films and Solar Cells by Vapor Deposition. J. Mater. Chem. A 2016, 4, 6693-6713.

(8) Saidaminov, M. I.; Abdelhady, A. L.; Murali, B.; Alarousu, E.; Burlakov, V. M.; Peng, W.;

Dursun, I.; Wang, L.; He, Y.; Maculan, G.; Goriely, A.; Wu, T.; Mohammed, O. F.; Bakr, O. M. High-Quality Bulk Hybrid Perovskite Single Crystals within Minutes by Inverse 
Temperature Crystallization. Nat. Commun. 2015, 6, 7586.

(9) Xiao, M.; Huang, F.; Huang, W.; Dkhissi, Y.; Zhu, Y.; Etheridge, J.; Gray-Weale, A.; Bach, U.; Cheng, Y.-B.; Spiccia, L. A Fast Deposition-Crystallization Procedure for Highly Efficient Lead Iodide Perovskite Thin-Film Solar Cells. Angew. Chemie Int. Ed. 2014, 53, 9898-9903.

(10) Berhe, T. A.; Su, W.-N.; Chen, C.-H.; Pan, C.-J.; Cheng, J.-H.; Chen, H.-M.; Tsai, M.-C.; Chen, L.-Y.; Dubale, A. A.; Hwang, B.-J. Organometal Halide Perovskite Solar Cells: Degradation and Stability. Energy Environ. Sci. 2016, 9, 323-356.

(11) Niu, G.; Li, W.; Meng, F.; Wang, L.; Dong, H.; Qiu, Y. Study on the Stability of CH3NH3PbI3 Films and the Effect of Post-Modification by Aluminum Oxide in All-SolidState Hybrid Solar Cells. J. Mater. Chem. A 2014, 2, 705-710.

(12) Meitl, M. A.; Zhu, Z.-T.; Kumar, V.; Lee, K. J.; Feng, X.; Huang, Y. Y.; Adesida, I.; Nuzzo, R. G.; Rogers, J. A. Transfer Printing by Kinetic Control of Adhesion to an Elastomeric Stamp. Nat. Mater. 2006, 5, 33-38.

(13) Lasky, J. B. Wafer Bonding for Silicon-on-insulator Technologies. Appl. Phys. Lett. 1986, $48,78-80$.

(14) Stengl, R.; Tan, T.; Gösele, U. A Model for the Silicon Wafer Bonding Process. Jpn. J. Appl. Phys. 1989, 28, 1735-1741.

(15) Hegner, M.; Wagner, P.; Semenza, G. Ultralarge Atomically Flat Template-Stripped Au Surfaces for Scanning Probe Microscopy. Surf. Sci. 1993, 291, 39-46.

(16) Nagpal, P.; Lindquist, N. C.; Oh, S.-H.; Norris, D. J. Ultrasmooth Patterned Metals for Plasmonics and Metamaterials. Science 2009, 325, 594-597.

(17) Prins, F.; Kim, D. K.; Cui, J.; De Leo, E.; Spiegel, L. L.; McPeak, K. M.; Norris, D. J. Direct 
Patterning of Colloidal Quantum-Dot Thin Films for Enhanced and Spectrally Selective Out-Coupling of Emission. Nano Lett. 2017, 17, 1319-1325.

(18) Yoo, D.; Johnson, T. W.; Cherukulappurath, S.; Norris, D. J.; Oh, S.-H. Template-Stripped Tunable Plasmonic Devices on Stretchable and Rollable Substrates. ACS Nano 2015, 9, $10647-10654$.

(19) Winkler, J. M.; Rabouw, F. T.; Rossinelli, A.; Jayanti, S.; McPeak, K. M.; Kim, D. K.; le Feber, B.; Prins, F.; Norris, D. J. Room-Temperature Strong Coupling of CdSe Nanoplatelets and Plasmonic Hole Arrays. Nano Lett. 2019, 19, 108-115.

(20) Chen, S.; Roh, K.; Lee, J.; Chong, W. K.; Lu, Y.; Mathews, N.; Sum, T. C.; Nurmikko, A. A Photonic Crystal Laser from Solution Based Organo-Lead Iodide Perovskite Thin Films. ACS Nano 2016, 10, 3959-3967.

(21) Fujihara, T.; Terakawa, S.; Matsushima, T.; Qin, C.; Yahiro, M.; Adachi, C. Fabrication of High Coverage MASnI 3 Perovskite Films for Stable, Planar Heterojunction Solar Cells. J. Mater. Chem. C 2017, 5, 1121-1127.

(22) Zhang, H.; Wang, Y.; Wang, H.; Ma, M.; Dong, S.; Xu, Q. Influence of Drying Temperature on Morphology of MAPbI3 Thin Films and the Performance of Solar Cells. J. Alloys Compd. 2018, 773, 511-518.

(23) Liu, M.; Johnston, M. B.; Snaith, H. J. Efficient Planar Heterojunction Perovskite Solar Cells by Vapour Deposition. Nature 2013, 501, 395-398.

(24) Malinkiewicz, O.; Yella, A.; Lee, Y. H.; Espallargas, G. M.; Graetzel, M.; Nazeeruddin, M. K.; Bolink, H. J. Perovskite Solar Cells Employing Organic Charge-Transport Layers. Nat. Photonics 2014, 8, 128-132.

(25) McPeak, K. M.; Jayanti, S. V.; Kress, S. J. P.; Meyer, S.; Iotti, S.; Rossinelli, A.; Norris, D. 
J. Plasmonic Films Can Easily Be Better: Rules and Recipes. ACS Photonics 2015, 2, 326333.

(26) Chandra, A.; Anderson, G.; Melkote, S.; Gao, W.; Haitjema, H.; Wegener, K. Role of Surfaces and Interfaces in Solar Cell Manufacturing. CIRP Ann. - Manuf. Technol. 2014, $63,797-819$.

(27) Ebbesen, T. W.; Lezec, H. J.; Ghaemi, H. F.; Thio, T.; Wolff, P. A. Extraordinary Optical Transmission through Sub-Wavelength Hole Arrays. Nature 1998, 391, 667-669.

(28) Maier, S. A. Plasmonics: Fundamentals and Applications; Springer US, 2007.

(29) Im, H.; Lee, S. H.; Wittenberg, N. J.; Johnson, T. W.; Lindquist, N. C.; Nagpal, P.; Norris, D. J.; Oh, S. H. Template-Stripped Smooth Ag Nanohole Arrays with Silica Shells for Surface Plasmon Resonance Biosensing. ACS Nano 2011, 5, 6244-6253.

(30) Blanchard-Dionne, A.-P.; Meunier, M. Sensing with Periodic Nanohole Arrays. Adv. Opt. Photonics 2017, 9, 891.

(31) Lindquist, N. C.; Nagpal, P.; McPeak, K. M.; Norris, D. J.; Oh, S.-H. Engineering Metallic Nanostructures for Plasmonics and Nanophotonics. Reports Prog. Phys. 2012, 75, 036501.

(32) Wang, H.; Haroldson, R.; Balachandran, B.; Zakhidov, A.; Sohal, S.; Chan, J. Y.; Zakhidov, A.; Hu, W. Nanoimprinted Perovskite Nanograting Photodetector with Improved Efficiency. ACS Nano 2016, 10, 10921-10928.

(33) Makarov, S. V.; Milichko, V.; Ushakova, E. V.; Omelyanovich, M.; Cerdan Pasaran, A.; Haroldson, R.; Balachandran, B.; Wang, H.; Hu, W.; Kivshar, Y. S.; Zakhidov, A. A. Multifold Emission Enhancement in Nanoimprinted Hybrid Perovskite Metasurfaces. ACS Photonics 2017, 4, 728-735.

(34) Chun, D. H.; Choi, Y. J.; In, Y.; Nam, J. K.; Choi, Y. J.; Yun, S.; Kim, W.; Choi, D.; Kim, 
D.; Shin, H.; Cho, J. H.; Park J. H. Halide Perovskite Nanopillar Photodetector. ACS Nano 2018, 12, 8564-8571.

(35) Kim, G.; An, S.; Hyeong, S.-K.; Lee, S.-K.; Kim, M.; Shin, N. Perovskite Pattern Formation by Chemical Vapor Deposition Using Photolithographically Defined Templates. Chem. Mater. 2019, 31, 8212-8221.

(36) Pourdavoud, N.; Wang, S.; Mayer, A.; Hu, T.; Chen, Y.; Marianovich, A.; Kowalsky, W.; Heiderhoff, R.; Scheer, H.-C.; Riedl, T. Photonic Nanostructures Patterned by Thermal Nanoimprint Directly into Organo-Metal Halide Perovskites. Adv. Mater. 2017, 29, 1605003.

(37) Pourdavoud, N.; Mayer, A.; Buchmüller, M.; Brinkmann, K.; Häger, T.; Hu, T.; Heiderhoff, R.; Shutsko, I.; Görrn, P.; Chen, Y.; Scheer, H.-C.; Riedl, T. Distributed Feedback Lasers Based on MAPbBr3. Adv. Mater. Technol. 2018, 3, 1700253.

(38) Brittman, S.; Oener, S. Z.; Guo, K.; Āboliṇš, H.; Koenderink, A. F.; Garnett, E. C. Controlling Crystallization to Imprint Nanophotonic Structures into Halide Perovskites Using Soft Lithography. J. Mater. Chem. C 2017, 5, 8301-8307.

(39) Zhizhchenko, A.; Syubaev, S.; Berestennikov, A.; Yulin, A. V.; Porfirev, A.; Pushkarev, A.; Shishkin, I.; Golokhvast, K.; Bogdanov, A. A.; Zakhidov, A. A.; Kuchmizhak, A. A.; Kivshar, Y. S.; Makarov, S. V. Single-Mode Lasing from Imprinted Halide-Perovskite Microdisks. ACS Nano 2019, 13, 4140-4147. 


\section{Supporting Information for:}

\section{Template-Stripping of Perovskite Thin Films for Dry-Interfacing and Surface-Structuring}

Alexander C. Hernandez Oendra, Eva De Leo, Stefan M. Koepfli ${ }^{\S}$, Jan M. Winkler, Aurelio A. Rossinelli, Andreas Riedinger ${ }^{t}$, David J. Norris, and Ferry Prins ${ }^{\dagger^{* *}}$

Optical Materials Engineering Laboratory, Department of Mechanical and Process Engineering, ETH Zurich, Rämistrasse 101, 8092 Zürich Switzerland 


\section{S-1. Fabrication of $\mathrm{CH}_{3} \mathrm{NH}_{3} \mathrm{Br}$ thin films}

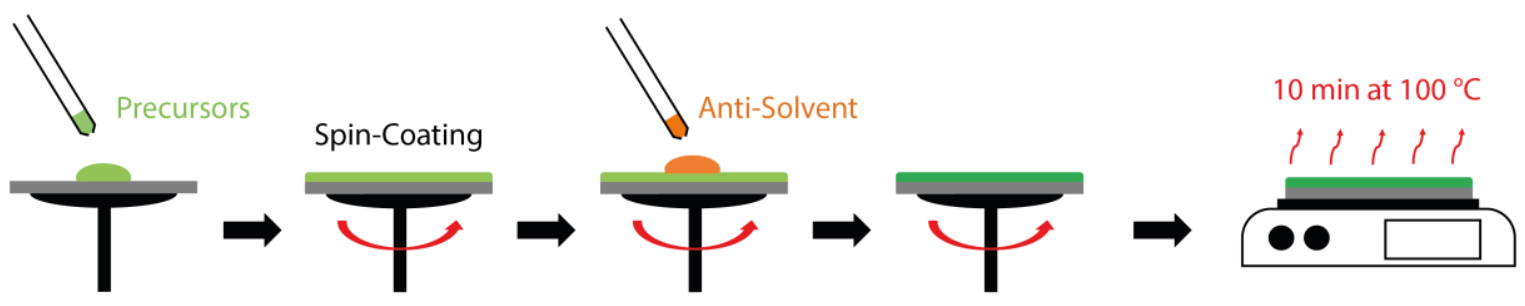

Figure S1. Schematics of the spin-coating procedure with applied anti-solvent dripping method.

A one-step spin-coating technique was implemented to fabricate the $\mathrm{CH}_{3} \mathrm{NH}_{3} \mathrm{PbBr}_{3}$ thin films. ${ }^{\mathrm{S} 1}$ The precursor solution was prepared by adding equimolar amounts of methylammonium bromide $\left(\mathrm{CH}_{3} \mathrm{NH}_{3} \mathrm{Br}\right)$ and lead bromide $\left(\mathrm{PbBr}_{2}\right)$ to dimethyl sulfoxide (DMSO) at a concentration of $25 \mathrm{wt}$ $\%$. To speed up the dissolution of the precursor, the solution was heated up to $70{ }^{\circ} \mathrm{C}$ for $60 \min$ and let cool down back to room temperature prior to further use.

The silicon templates, on which the perovskite thin films were deposited, were first cleaned by immersing them in piranha solution (1:1 ratio of sulfuric acid and hydrogen peroxide, $15 \mathrm{~min}$, Caution: Piranha solution reacts violently with solvents and other organic material). After rinsing them with deionized (DI) water, the templates were dried in an oven $\left(110^{\circ} \mathrm{C}, 2 \mathrm{~h}\right)$.

Shortly before the material was deposited, the template was treated with an ultraviolet-ozone cleaner (PSD, Novascan) for $30 \mathrm{~min}$. The UV-ozone treatment of the templates was not only performed to include another cleaning step but also to enhance the hydrophilicity of the template surface, which is beneficial to achieve a better wettability of the polar perovskite precursor solution on the substrate. The thin-film deposition was performed inside a glovebox by pipetting $50 \mu 1$ of the precursor solution onto the templates and spin-coating the sample at $2000 \mathrm{rpm}$ for $1 \mathrm{~min}$ with 
an initial acceleration of $2000 \mathrm{rpm} / \mathrm{s}$. Following the anti-solvent dripping procedure developed by Chen et al., $150 \mu \mathrm{m}$ of toluene was dropped onto the sample. ${ }^{\mathrm{S} 1}$

Using an anti-solvent dripping method yields perovskite films of enhanced uniformity compared to their spin-coated counterparts. ${ }^{\mathrm{S} 2}$ Finally, the sample is annealed for $10 \mathrm{~min}$ at $100{ }^{\circ} \mathrm{C}$ and stored in a glovebox until further use.

The thickness of the perovskite film can be varied by adjusting the concentration of the precursor solution. Decreasing the concentration down to $10 \mathrm{wt} \%$ allowed us to spin-coat and template-strip thin films with a thickness of only $25 \mathrm{~nm}$. 


\section{S-2. XRD measurements of $\mathrm{CH}_{3} \mathrm{NH}_{3} \mathrm{PbBr}_{3}$}

To verify the formation of the perovskite phase, we performed x-ray diffraction measurements. Figure S2 shows diffractograms of both the perovskite thin film and a perovskite thin film removed from the substrate as a powder. Both samples are compared to the diffractogram from a single-crystal X-ray diffraction ( $\mathrm{SCXRD)} \mathrm{measurement} \mathrm{of} \mathrm{a} \mathrm{CH}_{3} \mathrm{NH}_{3} \mathrm{PbBr}_{3}$ perovskite crystal at ambient pressure and temperature by Jaffe et al. ${ }^{\mathrm{S} 3}$ In contrast to the reference diffractogram, our thin film XRD measurement only features the diffractions associated with the (100) plane of the perovskite lattice and its higher orders. This suggests that the perovskite thin films crystallize predominantly with their (100) planes parallel to the surface of the template. We hypothesize that this is enabled due to the surface flatness. To verify this, powder XRD measurements were performed by scratching off perovskite films from their templates with a razor blade. The perovskite powder exhibits a more randomized distribution of the crystal planes, which leads to the appearance of the remaining peaks expected of a $\mathrm{CH}_{3} \mathrm{NH}_{3} \mathrm{PbBr}_{3}$ perovskite in the diffractogram as seen in the green line in Figure S2.

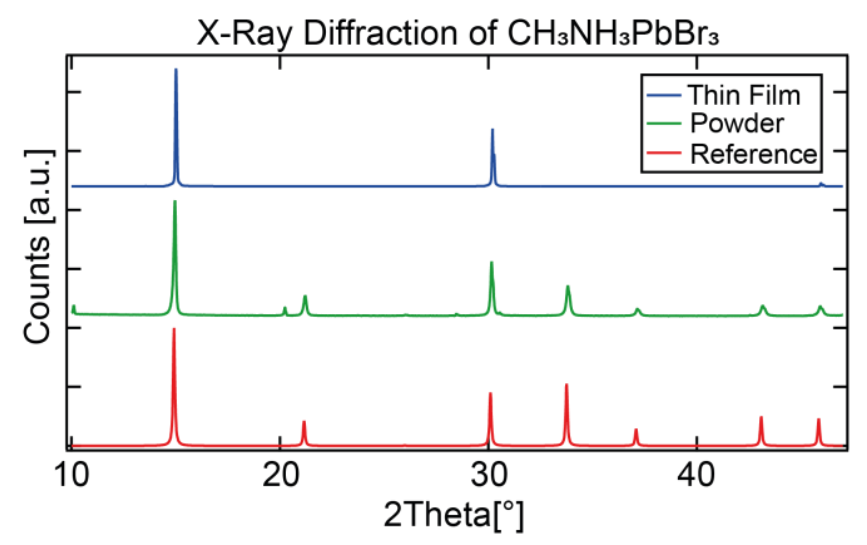

Figure S2. X-ray diffraction (XRD) patterns of the fabricated materials. The three different diffractograms are representing XRD measurements of a thin film (blue), perovskite powder 
produced by scratching off perovskite films from their silicon templates (green) and a single crystal X-ray diffraction (SCXRD) measurement published by Jaffe et al. ${ }^{\mathrm{S} 3}$ (red). 


\section{S-3. Functionalizing the surfaces of the silicon templates}

While template-stripping of flat interfaces is readily achieved using clean and pristine silicon templates, stripping from structured surfaces requires additional surface treatments to reduce the surface adhesion and prevent damage to small feature sizes during stripping. This means that on the one hand, the template needs to exhibit good wettability of the solvent used for deposition (DMSO in our case), and on the other hand requires relatively low adhesion to promote a clean stripping of the film from the structured template. For non-polar solvents, straightforward solutions have been demonstrated in which functionalization of the silicon template with low energy selfassembled monolayers (i.e. high water contact-angle) yields both good wettability, as well as low surface adhesion during stripping. ${ }^{\mathrm{S} 4}$ However, when using polar solvents such as DMSO, good wettability requires a high energy surface (small water contact-angle) which increases the surface adhesion and complicates the template-stripping procedure.

For template-stripping from flat interfaces, ozone-cleaned silicon templates were used with a characteristic water contact-angle $<30^{\circ}$, providing good wettability for DMSO and sufficiently low adhesion to yield high quality stripped films. Screening a variety of SAMs, carboxylic-acidtermination with a water contact-angle of $45^{\circ}$ was identified as having the best results for structured templates. The SAM treatment was modified from the method of Wassermann et al. ${ }^{\text {S5 }}$ In short, the silicon templates were first cleaned with piranha solution, which consisted of equal parts of sulfuric acid and hydrogen peroxide, for $15 \mathrm{~min}$. The templates were then submerged into DI water at $70{ }^{\circ} \mathrm{C}$ to rinse off the piranha solution and then dried under nitrogen gas. Subsequently, the templates were held over a beaker with DI water brought to a boil until condensate formed on their surface and immediately afterwards placed into the SAM precursor solution, which contained $150 \mu 1$ octenyltrichlorosilane, $300 \mu \mathrm{l}$ carbon tetrachloride, and $10 \mathrm{ml}$ bicyclohexyl. The templates 
stayed in the solution for $15 \mathrm{~min}$ and were subsequently sonicated in chloroform for $2 \mathrm{~min}$. This step was repeated 2 times to obtain a full coverage with the octenyltrichlorosilane-based SAMs. To interchange the vinyl termination with carboxylic acid functional groups, a permanganateperiodate oxidation was performed using a solution containing potassium permanganate $(0.5 \mathrm{mM})$, sodium periodate $(19.5 \mathrm{mM})$, and potassium carbonate $(1.8 \mathrm{mM})$ in DI water. The templates were submerged into the solution for $48 \mathrm{~h}$ and then rinsed with sodium bisulfite $(0.3 \mathrm{M})$, DI water, $10 \%$ hydrochloric acid, DI water and ethanol successively, which then yielded the carboxylic-acidterminated SAMs on our silicon templates. 


\section{S-4. Defects in $\mathrm{CH}_{3} \mathrm{NH}_{3} \mathrm{PbBr}_{3}$ thin film}

The surface of the template-stripped perovskite thin films can exhibit isolated indentations as seen in the AFM measurements in Figure S3a. These indentations most likely resulted during the template-stripping process and are not already present on the film after spin-coating. The formation of this kind of defects was presumably facilitated by introducing stresses into the film during the annealing step. Figure S3b shows a template-stripped perovskite film, which has not been annealed after spin-coating. The comparison between the two AFM measurements shows that the annealing step leads to an improved crystallinity of the film, which results in a smoother surface, but also introduces strain and stresses into the structure, which increases the chance of damaging the film while template-stripping.

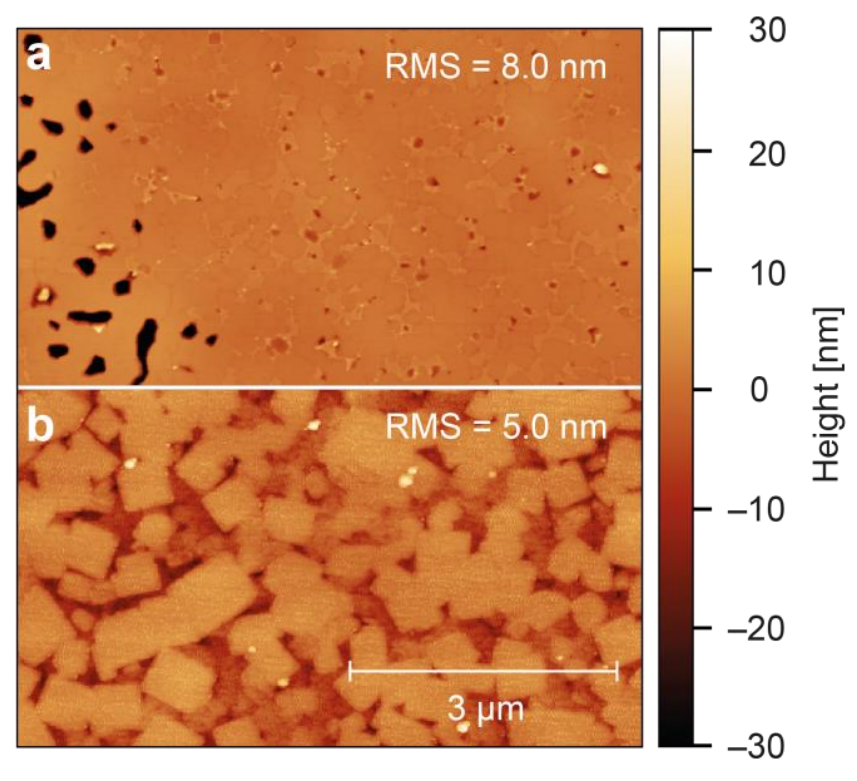

Figure S3. AFM measurements of perovskite thin films. (a) Template-stripped perovskite thin films can exhibit sinkhole like defects, which can lead to a massive increase of the RMS values. (b) If thin films are template-stripped without annealing the films after the spin-coating process, 
they exhibit lower crystallinity, resulting in rougher surfaces compared to their annealed counterparts. Both measurements were performed over a $7 \mathrm{x} 4 \mu \mathrm{m}^{2}$ area. 


\section{S-5. Structured $\mathrm{CH}_{3} \mathrm{NH}_{3} \mathrm{PbBr}_{3}$ thin films with submicrometer-sized features}

Using our method, we achieved fabrication of structured perovskite films with submicrometersized features. Figure S4 shows an SEM image of a perovskite film surface with pyramidal structures on its surface, which are individually $300 \mathrm{~nm}$ wide. The film exhibits significantly more defects compared to films with larger sized structural features (Figure 4). We attribute the higher density of defects in this size regime to the wettability issues specified in section S-3. We assume that not only the rupturing of structures during template-stripping, but also the inadequate filling of the template's structures with precursor solution during the spin-coating process, leads to defects in the final thin film. Improving the quality of nanometer-sized structures on templatestripped films could be achieved by further researching a suitable combination of spin-coating parameters and surface engineering of the templates.

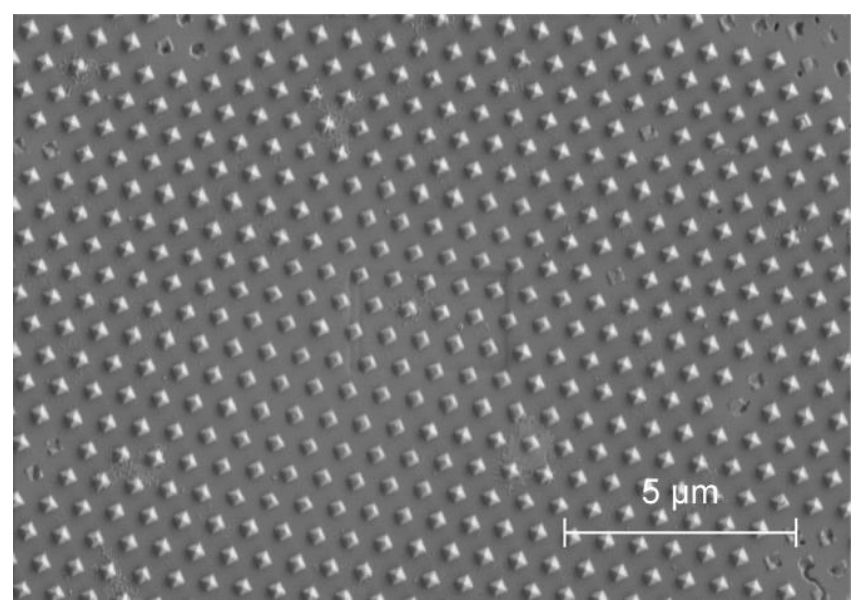

Figure S4. Template-stripped $\mathrm{CH}_{3} \mathrm{NH}_{3} \mathrm{PbBr}_{3}$ perovskite film with pyramid structure on its surface.

The edge length the individual pyramids is $300 \mathrm{~nm}$. 


\section{S-6. Plasmonic hole array fabrication}

The silver hole arrays for the perovskite interfacing were fabricated in two steps. For the templates, a four-inch diameter single-crystalline silicon $<100>$ wafer was diced into $2 \mathrm{~cm} \mathrm{x} 2 \mathrm{~cm}$ square chips. The chips were then cleaned with acetone for $3 \mathrm{~min}$ and then with isopropanol for another $3 \mathrm{~min}$ in an ultra-sonic bath. After a prebake at $180^{\circ} \mathrm{C}$ for $10 \mathrm{~min}$ and a quick cool-down, $280 \mathrm{~nm}$ of CSAR 62 resist (Allresist, AR-P 6200) were deposited by spin-coating at $2000 \mathrm{rpm}$ for $60 \mathrm{~s}$. The samples were then baked for $5 \mathrm{~min}$ at $150^{\circ} \mathrm{C}$. Patterning of the resist was performed using electron-beam lithography (Vistec, EBPG 520) and the exposed film was developed (1 min in Allresist, AR 600-546). Inductively coupled plasma deep reactive-ion etching (Oxford Plasmalab System 100, HBr, 150 s, $40 \mathrm{sccm}, 80 \mathrm{~W} \mathrm{RF}$ ) was then used to transfer the patterns onto the silicon chips. The remaining resist was then removed by oxygen plasma treatment $(100 \mathrm{~W}, 5$ min) and, subsequently, by placing the template in n-methyl-2-pyrrolidone (NMP) at $130^{\circ} \mathrm{C}$ for 2 $\mathrm{h}$ followed by sonication in acetone and isopropanol ( 5 minutes for each step). Finally, the chips were dried under nitrogen gas. The hole-array templates were then loaded into a thermal evaporator (Kurt J. Lesker, Nano36) to deposit a $200 \mathrm{~nm}$ thin silver film onto them. The source material is an Ag pellet (99.999\% purity, Kurt Lesker) which is placed in a tungsten dimple boat (Umicore). The chamber is evacuated to pressures in the order of $10^{-6}$ to $10^{-7}$ mbar and the deposition rate is set to $25 \AA / s$. 


\section{S-7. Estimation of the dispersion curves}

The dispersion curves shown in Figure 3 were calculated by evaluating the momentum matching equation for periodic gratings:

$$
\frac{2 \pi}{\lambda} n_{\text {eff }}=\left|\boldsymbol{k}_{\mathbf{0}} \sin (\theta)+m_{x} \frac{2 \pi}{\Lambda} \widehat{\boldsymbol{e}_{\boldsymbol{x}}}+m_{y} \frac{2 \pi}{\Lambda} \widehat{\boldsymbol{e}_{\boldsymbol{y}}}\right|
$$

where $k_{o} \sin (\theta)$ is the in-plane momentum of the free-space light, $\lambda$ the wavelength, $\Lambda$ the pitch between the holes, $\left[m_{x}, m_{y}\right]$ the diffraction orders in $\mathrm{x}$ and $\mathrm{y}$, respectively, $\widehat{\boldsymbol{e}}_{i}$ the basis vectors, and $n_{\text {eff }}$ the effective refractive index seen by the surface plasmon propagating at the metalperovskite interface. We only consider the fundamental modes $m=[ \pm 10],[0 \pm 1]$, since higherorder modes lie in the blue region of the spectrum. The effective refractive index is approximated as a second order polynomial of the form $f(\lambda)=a \lambda^{2}+b \lambda+c$ where the parameters are extracted from the high k-values of the measured dispersed k-spaces. The parameter $\mathrm{c}$ is used as a further fitting parameter. 

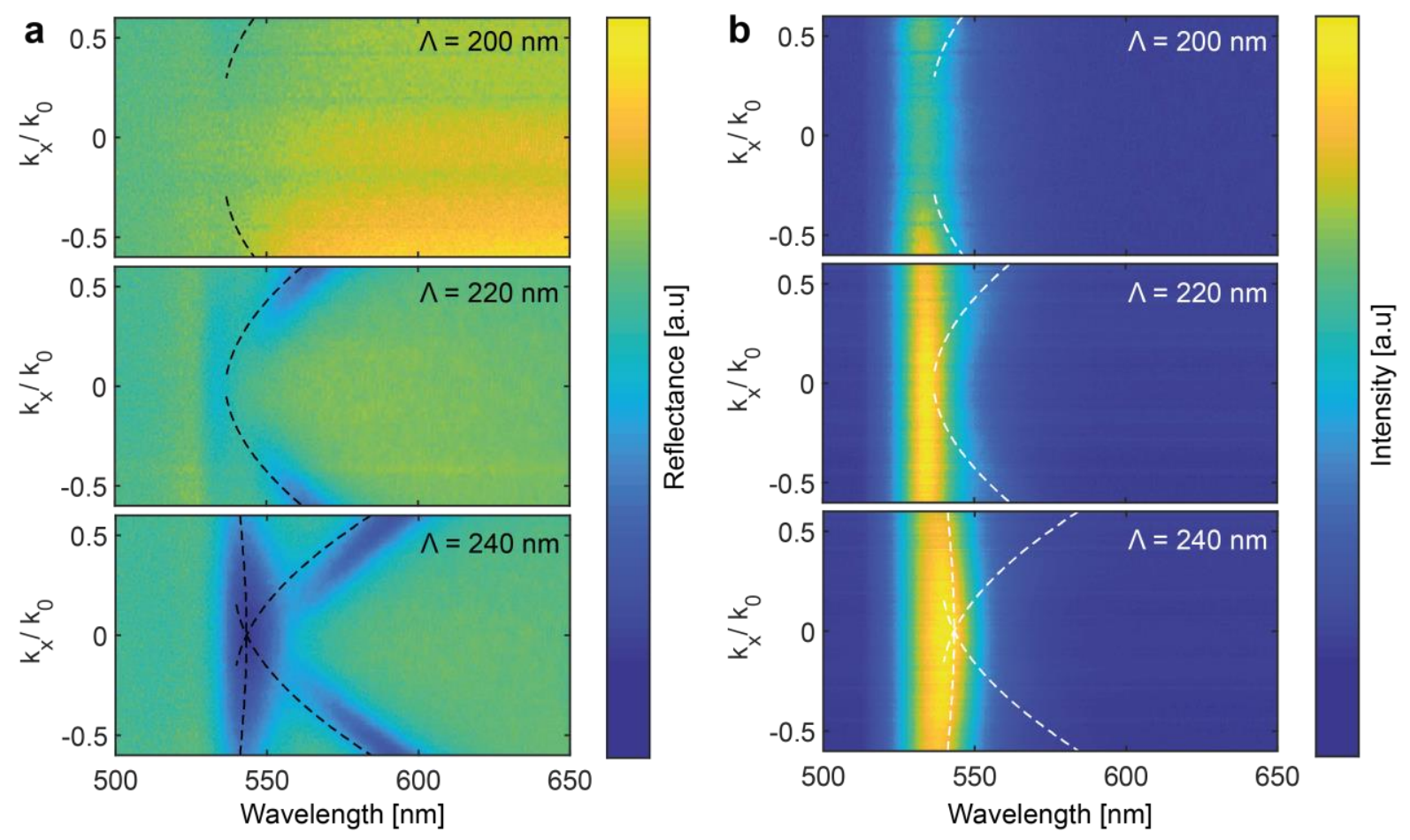

Figure S5. Dispersed k-spaces of the (a) reflection and (b) emission of $\mathrm{CH}_{3} \mathrm{NH}_{3} \mathrm{PbBr}_{3}$ perovskite films interfaced with plasmonic hole arrays. The pitch of the hole array is 200,220 , or $240 \mathrm{~nm}$ from top to bottom, respectively.

Figure S5 shows dispersed k-space plots of the reflectivity (Figure S5a) and emission (Figure S5b) from perovskite films on hole arrays. By varying the pitch $\Lambda$ of the hole array from 200 to $240 \mathrm{~nm}$, increased emission in the out-of-plane direction can be observed. This occurs when the crossing point of the dispersion branches of the hole array is spectrally aligned with the emission wavelength of the perovskite. The slight mismatch between the calculated dispersion curves and the measurements can be explained by the large change of the effective refractive index close to the absorption line of the perovskite thin film, which is not considered in the model.

The increased emissivity of the perovskite on the hole arrays could be due to the decreased quenching by the silver on the hole arrays. However, with increasing pitch length, the relative hole 
size is decreasing. Specifically, between a pitch of $200 \mathrm{~nm}$ and $240 \mathrm{~nm}$ the fill factor decreases from 0.3 to 0.27 . This leads to an increase of the surface area covered with silver with increasing pitch, which should lead to more quenching on hole arrays with longer pitches compared to the hole arrays with shorter pitches. Despite this counteracting effect, we observed an enhancement of the emission due to the coupling with resonant hole array structures.

The model allows for good prediction of dispersion curves also for hole arrays with pitches leading to the crossing of the dispersion branches at wavelengths far from the perovskite emission (occurring around $535 \mathrm{~nm}$ ). These measured and calculated absorption dispersion bands far from the perovskite absorption are shown in Figure S6.

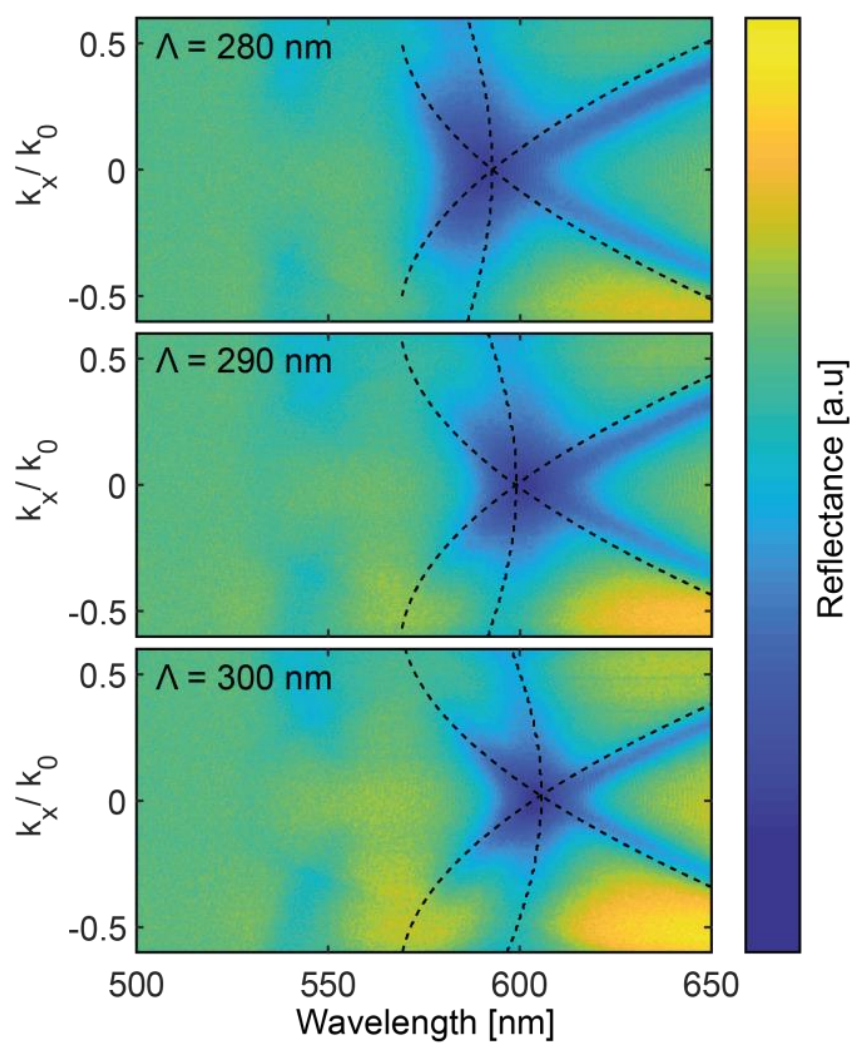


Figure S6. Dispersed k-space plots of $\mathrm{CH}_{3} \mathrm{NH}_{3} \mathrm{PbBr}_{3}$ perovskite films interfaced with off-resonant plasmonic hole arrays. The pitch of the hole array is 280,290 , or $300 \mathrm{~nm}$ from top to bottom, respectively. 


\section{S-8. Halide Diffusion at Perovskite/Perovskite Interface}

The quality of the interface and therefore especially the continuous contact between the perovskite and the desired surface is of utmost importance for the feasibility of our technique. While the vanishing of interference fringes at the interface as described in Figure 3 of the main text provides us with optical evidence of continuous contact between the surfaces, we explored the ion migration over an interface to reinforce our claims. We investigated the halide migration at an interface formed with two template-stripped perovskite films (Figure $\mathbf{S 7 a}$ ), $\mathrm{CH}_{3} \mathrm{NH}_{3} \mathrm{PbI}_{3}$ and $\mathrm{CH}_{3} \mathrm{NH}_{3} \mathrm{PbBr}_{3}$, by looking at the change of its absorption spectra over time. The $\mathrm{CH}_{3} \mathrm{NH}_{3} \mathrm{PbI}_{3}$ perovskite thin film was fabricated with the same procedure as the $\mathrm{CH}_{3} \mathrm{NH}_{3} \mathrm{PbBr}_{3}$ perovskite by using methylammonium iodide $\left(\mathrm{CH}_{3} \mathrm{NH}_{3} \mathrm{I}\right)$ and lead iodide $\left(\mathrm{PbI}_{2}\right)$ as the precursors. Templatestripping of both $\mathrm{CH}_{3} \mathrm{NH}_{3} \mathrm{PbI}_{3}$ and $\mathrm{CH}_{3} \mathrm{NH}_{3} \mathrm{PbBr}_{3}$ thin films was performed as described in the methods section. To create the interface, a template-stripped free-standing $\mathrm{CH}_{3} \mathrm{NH}_{3} \mathrm{PbBr}_{3}$ film (yellow circle on the left in Figure S7a) was pressed against a template-stripped $\mathrm{CH}_{3} \mathrm{NH}_{3} \mathrm{PbI}_{3}$ film (dark orange circle on the right in Figure S7a) until a transparent interface was visible.

As seen in Figure S7b, the interface initially shows the absorption peaks correlating to an exciton in $\mathrm{CH}_{3} \mathrm{NH}_{3} \mathrm{PbBr}_{3}$ at $530 \mathrm{~nm}$ and in $\mathrm{CH}_{3} \mathrm{NH}_{3} \mathrm{PbI}_{3}$ at $760 \mathrm{~nm}$. As time progresses, these two features decrease in intensity and shift towards each other energetically. This indicates a migration of halide ions over the interface leading to the buildup of a mixed halide perovskite zone. After 4 days, we observed the complete loss of the mono-halide perovskite absorption features and the evolution of an absorption feature around $640 \mathrm{~nm}$ correlating to the expected exciton peak in equally mixed iodine/bromine perovskite thin film. This was verified by measuring the absorption spectra of template-stripped perovskite thin films of different halide compositions. Figure S7c compares the spectra for the $100 \%$ iodine and the $100 \%$ bromide perovskites, with the spectrum of a $50 / 50$ 
iodine/bromine mixture fabricated by using equimolar amounts of the precursor salts. We conclude that the halide migration over the interface results in a homogeneous distribution of the halides over both films, which also confirms the continuous contact of both films and a high-quality interface.
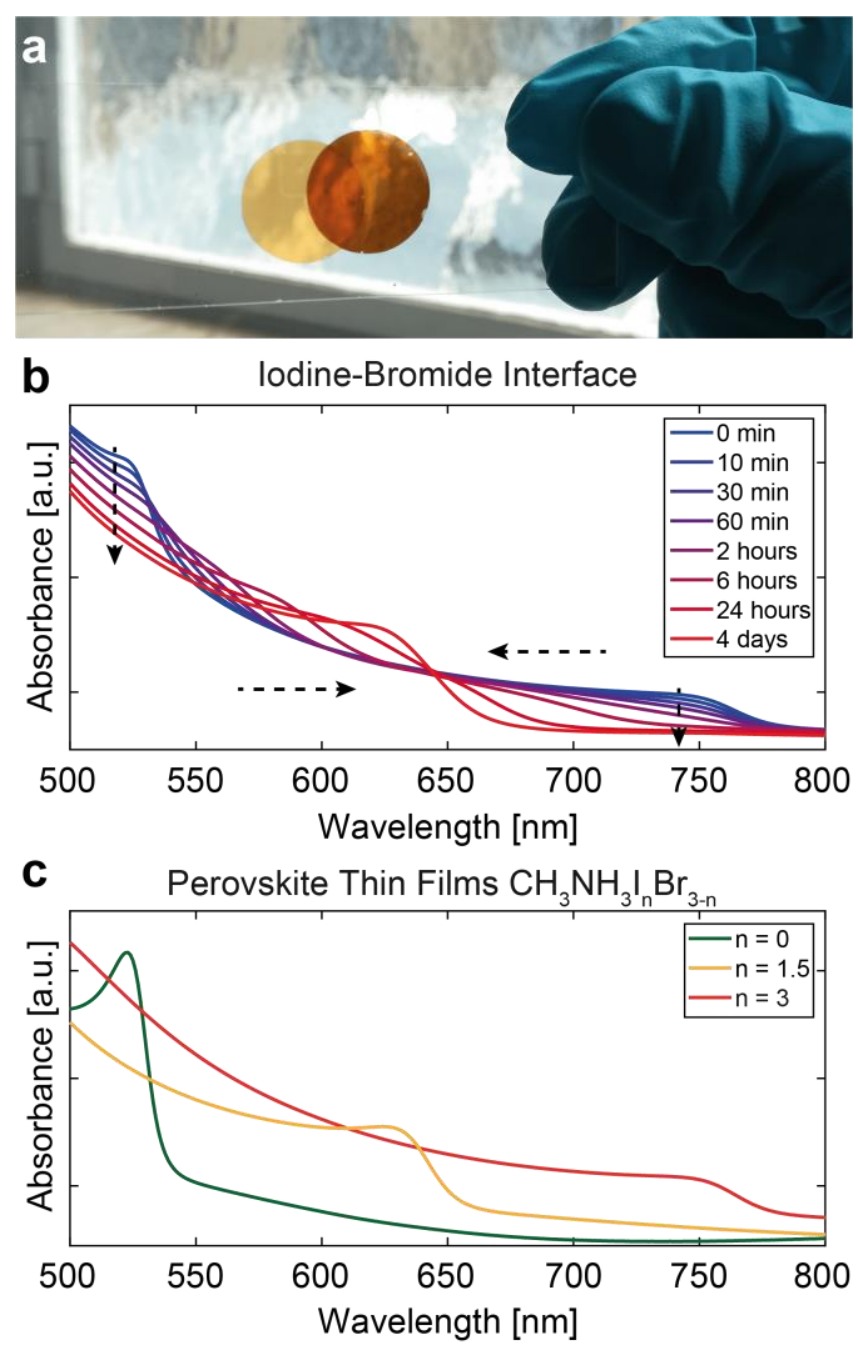

Figure S7. (a) An interface of template-stripped $\mathrm{CH}_{3} \mathrm{NH}_{3} \mathrm{PbI}_{3} / \mathrm{CH}_{3} \mathrm{NH}_{3} \mathrm{PbBr}_{3}$ perovskite thin films. (b) Halide migration across the interface revealed by time-dependent absorption spectra. (c) Absorption spectra of as-prepared template-stripped perovskite thin films with different halide compositions. 


\section{AUTHOR INFORMATION}

\section{Corresponding Author}

* E-mail: ferry.prins@uam.es (FP)

\section{SUPPORTING REFERENCES}

(S1) Chen, S.; Roh, K.; Lee, J.; Chong, W. K.; Lu, Y.; Mathews, N.; Sum, T. C.; Nurmikko, A. A Photonic Crystal Laser from Solution Based Organo-Lead Iodide Perovskite Thin Films. ACS Nano 2016, 10, 3959-3967.

(S2) Jeon, N. J.; Noh, J. H.; Kim, Y. C.; Yang, W. S.; Ryu, S.; Seok, S. Il. Solvent Engineering for High-Performance Inorganic-Organic Hybrid Perovskite Solar Cells. Nat. Mater. 2014, 13, 897-903.

(S3) Jaffe, A.; Lin, Y.; Beavers, C. M.; Voss, J.; Mao, W. L.; Karunadasa, H. I. High-Pressure Single-Crystal Structures of 3D Lead-Halide Hybrid Perovskites and Pressure Effects on Their Electronic and Optical Properties. ACS Cent. Sci. 2016, 2, 201-209.

(S4) Prins, F.; Kim, D. K.; Cui, J.; De Leo, E.; Spiegel, L. L.; McPeak, K. M.; Norris, D. J. Direct Patterning of Colloidal Quantum-Dot Thin Films for Enhanced and Spectrally Selective Out-Coupling of Emission. Nano Lett. 2017, 17, 1319-1325.

(S5) Wasserman, S. R.; Tao, Y. T.; Whitesides, G. M. Structure and Reactivity of Alkylsiloxane Monolayers Formed by Reaction of Alkyltrichlorosilanes on Silicon Substrates. Langmuir 1989, 5, 1074-1087. 